\title{
Clinical Findings and Autopsy of a Preterm Infant with Breast Milk-Acquired Cytomegalovirus Infection
}

\author{
Lopes Anne-Aurélie ${ }^{1} \quad$ Belhabri Souad, MD² Karaoui Leila, MD² \\ ${ }^{1}$ Faculté de Médecine Pierre et Marie Curie, Paris, France \\ 2 Service de Réanimation Néonatale, Centre Hospitalier de Meaux, \\ Meaux, France \\ Address for correspondence Lopes Anne-Aurélie, Faculté de Médecine \\ Pierre et Marie Curie, 15 Rue de l'Ecole de Médecine, 75006 Paris, \\ France (e-mail: anne-aurelie@club-internet.fr).
}

Am J Perinatol Rep 2016;6:e198-e202.

Abstract
Keywords
- neonate
- postnatal CMV
infection
- breast milk-acquired
infection
- autopsy

Background Nonpasteurized, nonfrozen, fresh breast milk from mothers with positive cytomegalovirus (CMV) serology was initially contraindicated to very low-birth-weight infants because of the risk of milk-acquired CMV infection. Recently, the severity of this infection was increasingly discussed and the international guidelines now differ. Since 2012, the American Academy of Pediatrics has recommended nutrition through raw breast milk for all preterm infants.

Case We report the case of an infant born prematurely at 27 weeks and 4 days and fed with raw breastmilk from day 12 of life (D12). He presented with a late-onset of CMV infection from D39. The CMV polymerase chain reaction (PCR), negative on D3, was strongly positive on D49, as well as the PCR on breast milk. He had CMV-specific immunoglobulin (g) M while his mother had only CMV-specific IgG. On D52, he deteriorated further with septic shock, and a fatal cardiac arrest on D54. His twin presented an asymptomatic CMV infection. The autopsy and histological examination showed evidence of numerous organ damage caused by CMV (with differences compared with congenital infection) but no evidence of bacterial infection.

Conclusion Although rare, postnatal CMV infections transmitted by raw breast milk given to very low-birthweight infants can have dramatic consequences.
In neonatal units, breastfeeding is a crucial part in the management of preterm infants. Its short- and long-term medical benefits were largely documented, as its immunological and nutritional values with implications for neurological development (via very long chain fatty acids and antioxidants), its earnings on digestive functions (with better absorption and a lower risk of necrotizing enterocolitis [NEC]) and its efficiency to decrease the incidence of infectious diseases. ${ }^{1}$ However, some infections, such as cytomegalovirus (CMV), can also be transmitted through breast milk.

The severity of congenital CMV infection is not discussed, but the knowledge about the severity of postnatal infections has evolved. First, feared because of a high morbidity and mortality, they were linked to transfusion-acquired CMV infections in infants of seronegative mothers, not protected by maternal antibodies. ${ }^{1,2}$ Since, preterm infants receive blood from a donor with negative CMV serostatus and the main source of CMV transmission is via raw breast milk from mothers with a positive-CMV serostatus. The CMV reactivation in breast milk is around $100 \%^{1,3}$ These infections are common, with negligible morbidity in term infants. In preterm infants, however, postnatal CMV infections can be described as asymptomatic or symptomatic with mild or severe symptoms such as sepsis-like symptoms. ${ }^{1-4}$ No received

May 6, 2015

accepted after revision

September 15, 2015
DOI http://dx.doi.org/

$10.1055 / \mathrm{s}-0035-1566249$. ISSN 2157-6998.
Copyright $\odot 2016$ by Thieme Medical

Publishers, Inc., 333 Seventh Avenue, New York, NY 10001, USA. Tel: +1(212) 584-4662.
License terms

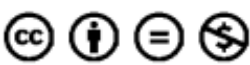


technique is actually available to preserve the nutritional value of breast milk and clear all viruses: pasteurization is effective to clear the virus, but significantly affect the nutritional and immunological values while freezing preserve the milk but do not clear all viruses. ${ }^{4}$

International guidelines agreed about the noncontraindication to breastfeeding for full-term infants whose mother is seropositive for CMV. On preterm infants, they agreed about a contraindication but it was increasingly criticized and the guidelines now differ on that subject. ${ }^{1,4-6}$ Since 2012, the American Academy of Pediatrics has recommended nutrition through raw breast milk for all preterm infants. According to them, the very low risk of severe breast milk-acquired CMV infection is not associated with long-term neurodevelopmental abnormalities and do not offset the many advantages of raw breast milk. In France, the guidelines retained to not feed infants that are $<32$ weeks of gestation or $<1,500 \mathrm{~g}$ with raw milk. We report a case of a very low-birth-weight infant (VLBWI) with a fatal breast milk-acquired CMV infection.

\section{Case Report}

A preterm boy was born from a monochorionic-diamniotic pregnancy with a twin-to-twin transfusion syndrome. At 27 weeks, he presented a severe inadequate intrauterine growth and his twin presented an absent umbilical artery diastolic flow with reversed ductus venosus a-wave flow and untrained heart. The treatment by intrauterine laser ablation of placenta vessels failed. The twins were born by cesarean section at 27 weeks and 4 days of gestation. His birth weight was $550 \mathrm{~g}$.

During the first month, he developed bronchopulmonary dysplasia (with invasive ventilation during 37 days), patent ductus arteriosus permanently closed by second drug treatment on the day of life (D) 9 and persistent anemia which required three transfusions on D0, D10, and D29. He was fed by nasogastric tube with his raw mother breast milk from D12 (29 weeks and 4 days, $690 \mathrm{~g}$ ). His mother's breast milk was unpasteurized, not frozen, stored at $4^{\circ} \mathrm{C}$, and given within 24 hours. He remained clinically stable.

From D39, he presented with bronchospasms associated with profound bradycardia and desaturation. On D40, food intolerance appeared, leading to the introduction of a new central catheter for total parenteral nutrition. He did not receive enteral nutrition again. On D45, he required an external cardiac massage with a new emergency intubation. Transfontanellar ultrasound and electroencephalography showed a global brain injury.

In this context, laboratory tests showed a persistent moderate inflammation (C-reactive protein around $12 \mathrm{mg} / \mathrm{L}$ and orosomucoid around $1.8 \mathrm{~g} / \mathrm{L}$ ) with thrombocytopenia (-Fig. 1) and elevated aspartate aminotransferase and alanine aminotransferase (around 170 and $65 \mathrm{IU} / \mathrm{L}$ from D39 to D55, respectively) but without bacteriological evidence. He did not improve with antibiotic therapy, which included cefotaxime, gentamicin, and vancomycin from D39 to D49. Viral searches, performed on D49, showed positive CMV viruria, positive blood CMV polymerase chain reaction (PCR) (300,000 copies/mL), with, on D52, presence of CMVspecific immunoglobulin M (IgM) (60 units $/ \mathrm{mL}$ ), and absence of CMV-specific IgG.

On D52, he deteriorated further with renal failure associated with septic shock may be caused by a secondary coinfection with methicillin-sensitive Staphylococcus aureus found in one hemoculture and a tracheal sample (C-reactive protein [CRP] $=209 \mathrm{mg} / \mathrm{L}$, platelet count of $30,000 / \mathrm{mm}^{3}$, decreased prothrombin to $43 \%$ but sterile central catheter, second hemoculture, and spinal cord fluid cultures). Without amelioration under antibiotics and supportive treatments, the infant remained with a hemodynamic instability and presented a fatal cardiac arrest on D54. Because of the short period of time between viral searches results and the deterioration leading to death, the antiviral treatment was neither discussed nor administrated.

The PCR done on maternal breast milk on D53 was positive too. The maternal CMV serologies done at the beginning of
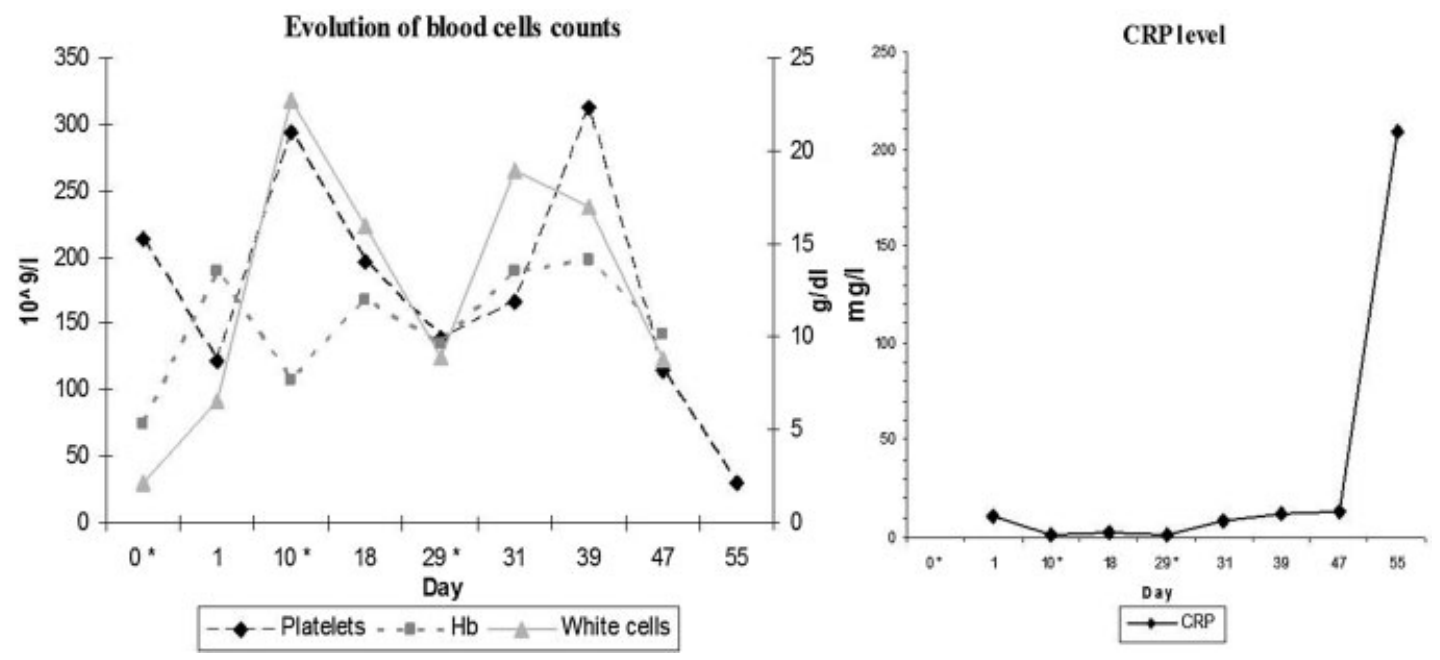

Fig. 1 Evolution of blood counts and CRP levels. *, blood transfusion; CRP, C-reactive protein; Day, day of life; Hb, hemoglobin. 
pregnancy, the day of delivery, and just after the death of the infant, showed virus-specific IgG without virus-specific IgM. The CMV PCR done on Guthrie test on D3 and on infant's urine on D4 was negative. The three transfusions were done according to a single-donor protocol with CMV-negative blood. After the death of the infant, the CMV PCR done on a residual sample of transfused blood was negative too.

During the same period, his twin developed bronchopulmonary dysplasia complicated by a patent ductus arteriosus closed by surgery after two drug treatment failures. Because of the twin-to-twin transfusion syndrome, he presented polycythemia and jaundice. He did not require a blood transfusion. As his twin, he was fed with raw breast milk by nasogastric tube from D12 (29 weeks and 4 days, $\sim 1,000$ g). He remained clinically stable. On D54, due to his brother's death, viral searches were performed. It showed positive CMV viruria with blood positive CMV-PCR $(40,000$ copies/mL) with CMV-specific IgM and IgG (7 IU/mL), without any biological and clinical symptom. The CMV PCR done retrospectively on Guthrie test (done on the D3) was negative.

The autopsy revealed numerous hemodynamic lesions (peritoneal and bilateral pleural effusions, hepatomegaly mottled with blood, renal necrosis, mucobloody stools, and two sites of meningeal hemorrhages). The histological examination showed a global depletion of lymphocytes. In the liver, hepatitis, cholestasis, and necrosis were seen. Typical "owl eyes" inclusions, specific signs of CMV, were seen in lungs (pneumocytes and lung macrophages, reflecting a CMV pneumonia) (-Fig. 2), in tubular cells of kidneys with large necrosis destroying all tubular structures, in the liver, in the endothelium of the pancreas, and in capillaries of inflammatory adrenal glands. The stomach and the intestinal tract showed inflammation and large necrosis with cytonuclear dystrophy in the epithelium, evocative of CMV infection (-Fig. 3). The brain showed total inflammation associated with foci of neuronophagy in the basal ganglia (mainly thalamus) and the cerebral trunk, suggesting of CMV encephalitis. No cultures from liver, heart, lung, kidneys, spleen, meninges, or the choroids plexus showed the presence of $S$. aureus.

\section{Discussion}

In recent years, more and more cases of postnatal CMV infections with sepsis-like symptoms have been reported. In the literature, all reports of sepsis-like symptoms and multiple organ involvement concerned VLBWI. The clinical course was similar to our case with pneumonia and bronchospasms (which often required emergency intubation), hepatitis, cholestasis, hepatosplenomegaly, colitis, and disseminated intravascular coagulation requiring supportive treatment. In a majority of cases, treatment with ganciclovir was used and they subsequently recovered. ${ }^{7-10}$ Some cases showed a transient hearing impairment or a possibility of neurological delay. One case had moderate white abnormalities on the MRI, another required extracorporeal membrane oxygenation and was discharged with a tracheostomy on home mechanical ventilation ${ }^{7-10}$ and another was a case of fatal postnatal CMV infection in a VLBWI. In this one too, ganciclovir was used and the clinical course differed from the others, in the presence of a renal failure, ${ }^{7}$ such as in our case.

In our department, before this case and even if it was not compliant with the French recommendations, all the preterm infants were fed with raw breast milk. It was done considering the incomparable nutritional and immunological values of raw breast milk, its documented short- and long-term

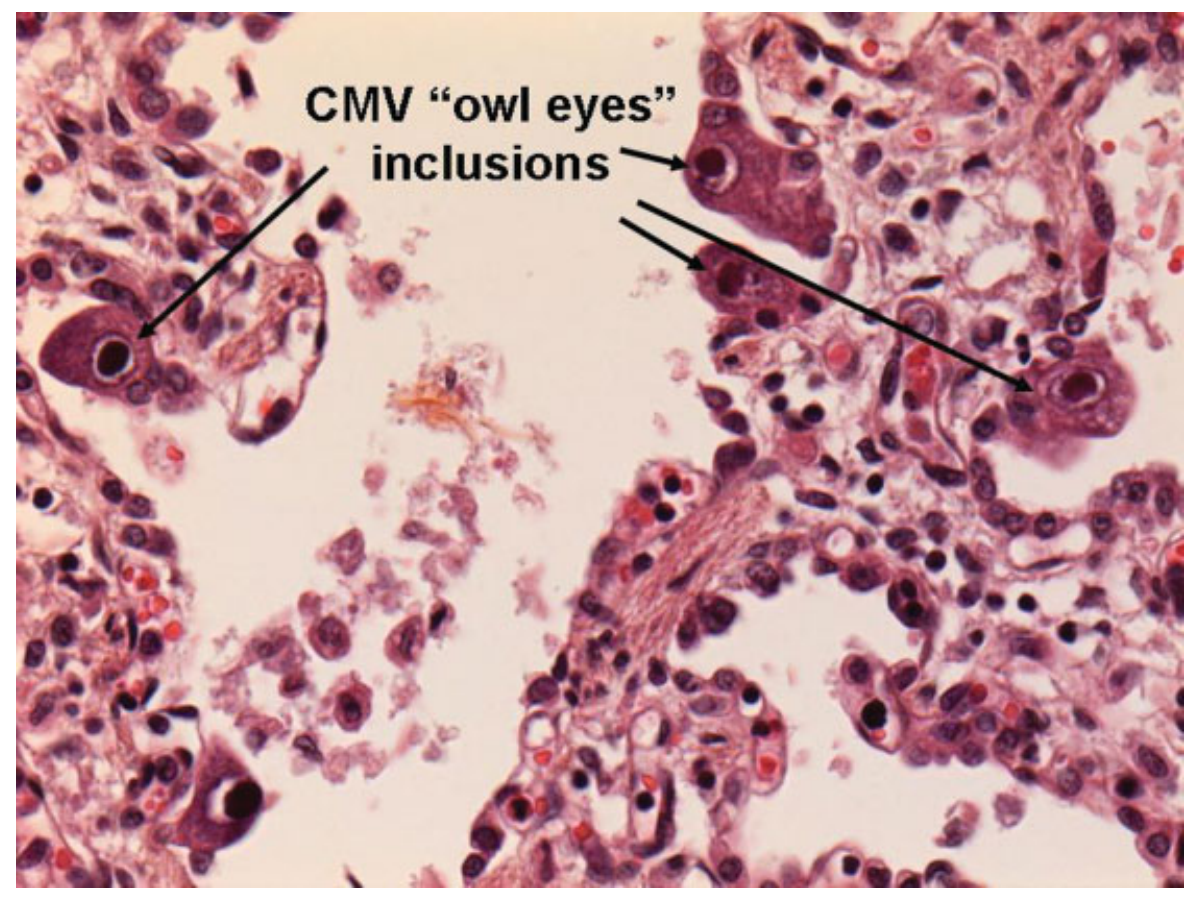

Fig. 2 Pneumocytes with typical CMV "owl eyes" inclusions. CMV, cytomegalovirus. 


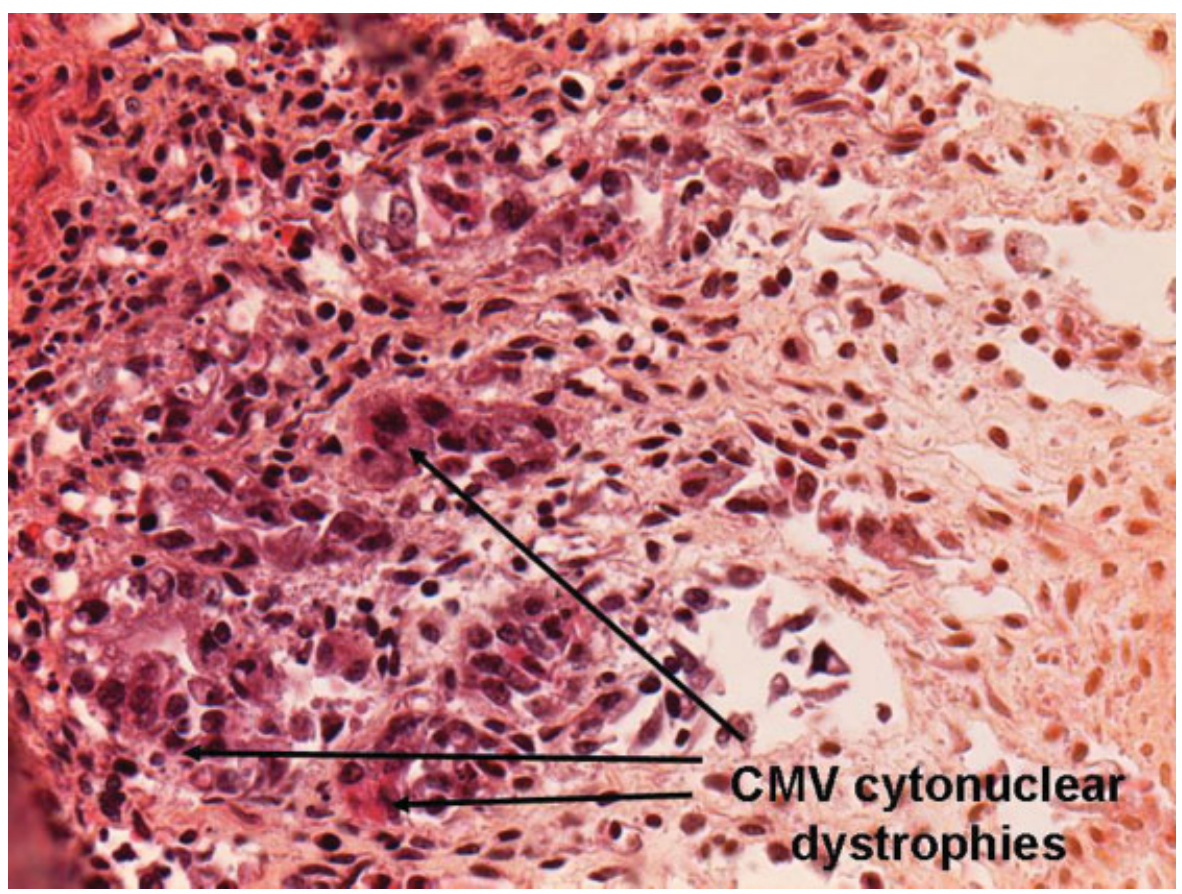

Fig. 3 Gastric glands with cytonuclear dystrophies typical of CMV infection. CMV, cytomegalovirus.

medical and neurodevelopmental advantages, the absence of available technique to destroy all viruses and preserve the milk, the absence of any symptomatic postnatal CMV infection in our department, and the positive psychological impact on mothers. The parents were informed about the benefits of raw breast milk and the risk of bacterial infections, prevented by milk samples for bacterial research, but the risk of viral infections (not specifically CMV) was briefly mentioned.

After this case, it was decided to feed infants with a weight under $1,500 \mathrm{~g}$ with pasteurized women milk from the milk bank and to feed them with their mother's milk (frozen or raw) after they reached $1,500 \mathrm{~g}$ : a protocol was written.

For the time being, it seems that there is no predicting factor for CMV infections or its severity, but the population concerned seems to be the VLBWI with a growing number of reported cases about severe breast milk-acquired CMV infection. In our mind, even if the French contraindication of raw breast milk before 32 weeks or $1,500 \mathrm{~g}$ appears to be exaggerated, its recommendation in all preterm infants has to be discussed again. The preferable way to feed a preterm infant could not be found only on the term and weight, but required careful thought on his health status, including his comorbidities and weaknesses identified at a certain point, to balance the benefits and risks of raw milk. In our opinion, an individual decision should be preferred to general guidelines. However, the discussion is complicated by the fact that, in our cases, his twin was also fed with raw breast milk, but developed an asymptomatic CMV infection and did not present significant difference to the patient, except the transfused anemia and their weight.

Nonetheless, some caveats need to be considered. No genotyping of the CMV was done on infant's or mother's samples to prove the transmission. Recent studies about postnatal CMV infections showed that some could be attributed to a failure to prevent transfusion transmitted-CMV due to donors in the window phase of infection..$^{11}$ However, in our case, the transfusions were done according to a single-donor protocol with CMV-negative blood and the CMV PCR done on a residual sample of transfused blood was negative. Moreover, his twin also had a postnatal CMV infection and did not receive blood transfusions. Another caveat is that we did not perform genetic analyses to rule out immunodeficiency diseases. On congenital infection reports, immunodeficiency was observed in $11 \%$ of cases, ${ }^{12}$ but our case was about a monochorionic-diamniotic pregnancy and his twin is now doing well, without any suspicion of immunodeficiency.

The low mortality rate explains the absence of autopsy in the literatures. To our knowledge, this is the first autopsy report about an infant with postnatal CMV infection. In congenital infection, the most commonly involved organs are the lungs and kidneys (with the presence of "owl eyes" inclusions in pneumocytes and tubule epithelium with necrosis), followed by liver (hepatitis, cholestasis, and necrosis), thymus, and brain. Seen less frequently are the lesions of heart, pancreas, and adrenal glands. The "owl eyes" inclusions can be present in all organs and were found predominantly in the epithelium and very few in the endothelium. There is no description of the digestive tract or other organ involvement in congenital infection. ${ }^{12-14}$

In our case, the same organs are involved with typical "owl eyes" inclusions in lungs, kidneys, and liver and lesions, suggesting a CMV infection in brain, pancreas, and adrenal gland. In the pancreas and adrenal gland, the inclusions are situated in the endothelium and very few in the epithelium. Contrary to congenital infection, the digestive tract was involved and the lesions are the same as those seen in adult 
infections and could be a sign of contamination via the digestive tract.

Previous reports described that CMV infection is straightforward when typical "owl eyes" inclusions are present ${ }^{12,14}$ and that the infection tended to be systemic when "owl eyes" inclusions are present in the liver, ${ }^{12}$ therefore, we can assume that it was a systemic CMV infection. However, to confirm that the lesions in brain, pancreas, adrenal glands, and digestive tract are due to the CMV, immunohistochemical studies using antiCMV antibody are necessary and were, unfortunately, not done.

Enteritis often occurs in immunocompromised adults and feeding intolerance is a described manifestation of CMV infection in infants. It can mimic NEC and can require surgical intervention. $^{2,15}$ In our case, the histological examination revealed inflammation and large necrosis of the digestive tract, which are also typical lesions of the NEC. The pathogenesis of digestive tract involvement in CMV infection and its link with NEC are still unclear. Previous studies about the clinical manifestation of postnatal breast-milk acquired CMV infection did not report $\mathrm{NEC}^{6,16}$ or reported it without no significant difference with the group of preterm infant without postnatal CMV infections. ${ }^{5,17}$

In postnatal infections, we can see a global depletion of lymphocytes without important inflammation: in congenital infection, it has been attributed to an immature cellular immune reaction associated with prematurity and showed that cellular damage is mostly caused by a direct cytopathic effect of CMV rather than immunologic reaction. ${ }^{12}$ We can assume that it is the same physiopathology in postnatal infection of VLBWI.

CMV infection is commonly associated with neutropenia, but there is no increased risk of bacterial infection described in the literature. $^{6-10}$ In our case, the postnatal CMV infection was associated with a secondary co-infection of methicillin-sensitive staphylococcus aureus. On the day of clinical deterioration, a positive hemoculture and a positive trachea sample with an elevation of CRP could not be explained only by the CMV infection. However, one of the hemoculture done the same day as the spinal cord fluid and the catheter cultures, was sterile and there is no bacterial evidence in the different organs cultures done during the autopsy. The damage found in the organs was characteristic or suggesting a CMV infection and the infant had a high level of viremia. This seemed to show that the CMV infection was the proximate cause of his deterioration.

In developed countries, the number of preterm infants and VLBWI is increasing. Given the CMV reactivation in breast milk, the incidence of CMV in women general population, and the risk of symptomatic infection in VLBWI, CMV must be considered in all VLBWI presenting with late-onset neonatal sepsis. For their optimal management, the development of an available technique to destroy all viruses and preserve the values of the milk would be a perfect solution, however, for now, an epidemiologic monitoring to identify cases is necessary to determine what the actual risk is and what the necessary and adequate preventive measures are.

\section{Conflict of Interest}

We report no conflict of interest.

\section{Acknowledgments}

The authors thank Dr. Rambaud and the Department of pathological anatomy of Raymond Poincarré Hospital, Paris for the autopsy and histological examination results and photographs.

\section{References}

1 Kurath S, Halwachs-Baumann G, Müller W, Resch B. Transmission of cytomegalovirus via breast milk to the prematurely born infant: a systematic review. Clin Microbiol Infect 2010;16(8):1172-1178

2 Vochem M, Hamprecht K, Jahn G, Speer CP. Transmission of cytomegalovirus to preterm infants through breast milk. Pediatr Infect Dis J 1998;17(1):53-58

3 Hamprecht K, Maschmann J, Vochem M, Dietz K, Speer CP, Jahn G. Epidemiology of transmission of cytomegalovirus from mother to preterm infant by breastfeeding. Lancet 2001;357(9255):513-518

4 Lanzieri TM, Dollard SC, Josephson CD, Schmid DS, Bialek SR. Breast milk-acquired cytomegalovirus infection and disease in VLBW and premature infants. Pediatrics 2013;131(6): e1937-e1945

5 Neuberger P, Hamprecht K, Vochem M, et al. Case-control study of symptoms and neonatal outcome of human milk-transmitted cytomegalovirus infection in premature infants. J Pediatr 2006; 148(3):326-331

6 Miron D, Brosilow S, Felszer K, et al. Incidence and clinical manifestations of breast milk-acquired Cytomegalovirus infection in low birth weight infants. J Perinatol 2005;25(5):299-303

7 Hamele M, Flanagan R, Loomis CA, Stevens T, Fairchok MP. Severe morbidity and mortality with breast milk associated cytomegalovirus infection. Pediatr Infect Dis J 2010;29(1):84-86

8 Fischer C, Meylan P, Bickle Graz M, et al. Severe postnatally acquired cytomegalovirus infection presenting with colitis, pneumonitis and sepsis-like syndrome in an extremely low birthweight infant. Neonatology 2010;97(4):339-345

9 Takahashi R, Tagawa M, Sanjo M, et al. Severe postnatal cytomegalovirus infection in a very premature infant. Neonatology 2007; 92(4):236-239

10 Okulu E, Akin IM, Atasay B, Ciftçi E, Arsan S, Türmen T. Severe postnatal cytomegalovirus infection with multisystem involvement in an extremely low birth weight infant. J Perinatol 2012; 32(1):72-74

11 Josephson CD, Caliendo AM, Easley KA, et al. Blood transfusion and breast milk transmission of cytomegalovirus in very low-birthweight infants: a prospective cohort study. JAMA Pediatr 2014; 168(11):1054-1062

12 Kosai K, Kage M, Kojiro M. Clinicopathological study of liver involvement in cytomegalovirus infection in infant autopsy cases. J Gastroenterol Hepatol 1991;6(6):603-608

13 Peña-Alonso R, Navarrete-Navarro S, Ramon-Garcia G, HernandezMote R, Rodriguez-Jurado R. Cytomegalovirus infection in children: frequency, anatomopathologic characteristics and underlying risk factors in 1618 autopsies. Arch Med Res 1996;27(1):25-30

14 Ko HM, Kim KS, Park JW, et al. Congenital cytomegalovirus infection: three autopsy case reports. J Korean Med Sci 2000; 15(3):337-342

15 Tengsupakul S, Birge ND, Bendel CM, et al. Asymptomatic DNAemia heralds CMV-associated NEC: case report, review, and rationale for preemption. Pediatrics 2013;132(5):e1428-e1434

16 Goelz R, Hamprecht K, Vochem M, et al. Muttermilchernährung der sehr unreifen Frühgeborenen von HCMV-seropositiven Müttern-Stellungnahme. Z Geburtshilfe Neonatol 2004;208(3): 118-121

17 Hamprecht K, Maschmann J, Jahn G, Poets CF, Goelz R. Cytomegalovirus transmission to preterm infants during lactation. J Clin Virol 2008;41(3):198-205 